\title{
The prevalence and management of rifampicin-resistant tubercu- losis among adults at Chris Hani Baragwanath Academic Hospital, Johannesburg, South Africa
}

\author{
R M Narsing, ${ }^{1,3}$ MB BCh, FCP; A S Karstaedt, ${ }^{2,3}$ MB BCh, MMed; F Sahid, ${ }^{2,3}$ MB BCh, FCP \\ ${ }^{1}$ Division of Pulmonology, Department of Internal Medicine, Chris Hani Baragwanath Academic Hospital, Johannesburg, South Africa \\ ${ }^{2}$ Division of Infectious Diseases, Department of Internal Medicine, Chris Hani Baragwanath Academic Hospital, Johannesburg, South Africa \\ ${ }^{3}$ School of Health Sciences, University of the Witwatersrand, Johannesburg, South Africa. \\ Corresponding author: R M Narsing (raj4065@yahoo.com)
}

\begin{abstract}
Background. Early effective management of drug-resistant tuberculosis (TB) is important for the patient, and for infection control. The Xpert MTB/RIF (Cepheid, USA) assay detects Mycobacterium tuberculosis DNA and the presence of rifampicin resistance.

Objective. To assess the prevalence and initial management of rifampicin-resistant pulmonary TB (PTB), confirmed by the Xpert MTB/ RIF assay, in hospitalised adult patients.

Methods. This retrospective descriptive study assessed adult patients from March 2011 to February 2013. Data was obtained from the National Health Laboratory Service database and patient records. Management comprised the submission of additional confirmatory sputum tests, initiation of appropriate anti-TB drug therapy, patient isolation, and proper referral.

Results. The prevalence of rifampicin resistance was $10.6 \%(n=77)$ of 729 positive assays. The initial management was assessed for 70 patients with complete records. However, of these 70 patients, 12 patients had been discharged and 5 patients had died prior to receiving their results. The management of the total cohort, and of the 53 remaining inpatients, was analysed separately. The overall confirmatory sputum submission rates were $76 \%, 60 \%, 60 \%$ and $26 \%$ for TB microscopy, Line Probe Assay, TB culture, and drug-susceptibility testing, respectively, and $87 \%, 72 \%, 68 \%$ and $30 \%$, respectively for the 53 remaining patients. Overall, $33 \%$ of patients received appropriate anti-TB treatment, $50 \%$ were isolated, and $49 \%$ were appropriately referred. For the 53 remaining patients, $43 \%$ received appropriate drug treatment, $66 \%$ were isolated, $64 \%$ were appropriately referred, and $19 \%$ were not referred. The inpatient mortality rate was $19 \%$.

Conclusions. Rifampicin-resistant TB prevalence in-hospital was more than double the national rate. The initial management of patients with rifampicin-resistant PTB was substandard. Submission of paired sputum samples and educating healthcare professionals and healthcare users are of paramount importance to improve the management of drug-resistant TB.
\end{abstract}

S Afr Respir J 2017;23(2):30-34. DOI:10.7196/SARJ.2017.v23i2.151

Tuberculosis (TB) causes a significant burden of disease worldwide, with $~ 9.6$ million cases and 1.5 million deaths in $2014 .{ }^{[1]}$ South Africa (SA) is ranked among the top six countries in the world as having the largest number of incident cases. ${ }^{[1,2]}$ Mycobacterium tuberculosis (MTB) is the organism responsible for TB infection and has been treated successfully for many decades. The emergence of drugresistant TB has complicated the management of the disease. Multidrug-resistant tuberculosis (MDR-TB) is defined as resistance to the two most effective first-line drugs, isoniazid (INH) and rifampicin (RIF). ${ }^{[3]}$ Of the new TB cases reported in 2013 and 2014, there were 480 000 cases of MDR-TB annually, ${ }^{[1,2,4]}$ with an estimated 6900 cases in SA in $2014 .{ }^{[4]}$ It was estimated that $3.3 \%$ of new TB cases were due to MDR-TB, whereas $20 \%$ of previously treated TB cases had presented with MDR-TB in 2014. ${ }^{[1]}$ Risk factors for the development of MDR-TB include previous exposure to anti-TB treatment, incomplete treatment regimens, poor patient compliance, lack of availability of adequate medication, co-infection with HIV, social barriers, lack of patient access to care, poorly coordinated management strategies from healthcare institutions and inadequate guidelines. ${ }^{[5-11]}$

In 2013, there were an estimated 2.1 million new HIV infections globally and 1.5 million new infections in the World Health
Organization (WHO) African region. ${ }^{[12]}$ Patients who are coinfected with HIV and TB are more likely to have rapid progression to active TB disease, have a higher likelihood of MDR-TB, and higher mortality. ${ }^{[4,13-17]}$ The incidence of HIV co-infection and TB is the highest in the African region, with about $80 \%$ of cases being co-infected. ${ }^{[2]}$ In sub-Saharan Africa there was an estimated $70 \%$ co-infection rate of drug-resistant TB and HIV.$^{[2,14]}$ Gandhi et al. ${ }^{[18]}$ demonstrated a $92 \%$ co-infection rate of MDR-TB and HIV in KwaZulu-Natal Province, SA.

Resistance to RIF usually occurs due to point mutations of the $r p o B$ gene, ${ }^{[3,19,20]}$ with more than $95 \%$ of mutations responsible for the development of RIF resistance occurring in this gene. ${ }^{[3,21]}$ In regions with a high probability of MDR-TB, such as sub-Saharan Africa, RIF resistance can be used as a reliable indicator for MDR-TB. ${ }^{[22]}$ The WHO has endorsed the use of molecular testing for the rapid diagnosis of TB and drug resistance. ${ }^{[23]}$ The Xpert MTB/RIF (Cepheid, USA) assay has been approved for use since December 2010. The assay detects MTB DNA and the presence of RIF resistance. ${ }^{[23]}$ Studies reviewing the effectiveness of the Xpert MTB/RIF assay found a sensitivity of $95 \%$ and specificities of $94-98 \%$ with smear-positive sputum and sensitivities of $55 \%$ and $67 \%$ in smear-negative sputum. ${ }^{[24,25]}$ The rapid 
result is an advantage when compared to the lengthier duration of conventional drug susceptibility testing (DST). ${ }^{[26]}$

In 2011, the SA Department of Health released guidelines for the management of drug-resistant TB. ${ }^{[27]}$ Following the result of RIFresistant MTB on the Xpert MTB/RIF assay, MDR-TB treatment should be initiated, confirmatory sputum specimens submitted, patients should be isolated and appropriately referred. Treatment should then be reviewed following laboratory confirmation of drug susceptibility. ${ }^{[27]}$ A combination of five effective chemotherapeutic agents are recommended for the treatment of MDR-TB. ${ }^{[28]}$ These include a fluoroquinolone (moxifloxacin or ofloxacin), a secondline aminoglycoside (kanamycin or amikacin), pyrazinamide, ethionamide and terizidone. ${ }^{[27]}$ Commitment from both the patient and the healthcare professional is essential to treat TB and drugresistance effectively in affected communities. Local studies have shown that patient compliance, delay in initiation of treatment, loss to follow-up, poor contact tracing, and early mortality still remain hindering factors in current treatment strategies. ${ }^{[29-31]}$ Ebonwu et al. ${ }^{[30]}$ found that only $63 \%$ of patients were initiated on MDR-TB treatment in Gauteng Province in 2011.

This study aimed to describe the prevalence and initial management of hospitalised adult patients with RIF-resistant pulmonary TB (PTB), as diagnosed by the Xpert MTB/RIF .

\section{Methods}

Study setting

Chris Hani Baragwanath Academic Hospital (CHBAH) is a tertiarylevel hospital with 702 adult medical beds that serves the community of Soweto and surrounding areas.

\section{Study design}

This retrospective descriptive study, based on record review, assessed adult patients with RIF-resistant PTB during a two-year period from March 2011 to February 2013. The National Health Laboratory Service (NHLS) provided a list of all patients with a positive Xpert MTB/RIF assay result. Patients included in the study were older than 14 years and were admitted to the adult medical wards at $\mathrm{CHBAH}$.

The prevalence of RIF resistance was calculated by dividing the number of positive RIF-resistant Xpert MTB/RIF assays by the total number of positive Xpert MTB/RIF assays (i.e. RIF-sensitive and RIF-resistant) submitted by adult patients at CHBAH during the 2-year period. RIF resistance was further subdivided into MDR-TB (resistance to both RIF and INH), RMR (resistance to RIF only) and unclassified (patients without confirmatory test submission). The management of patients was assessed based on submission of the confirmatory sputum samples (line probe assay (LPA), TB microscopy, TB culture, and DST), appropriate drug therapy (a fluoroquinolone, such as moxifloxacin or ofloxacin, a second-line aminoglycoside, such as kanamycin or amikacin, pyrazinamide, ethionamide, and terizidone), adequate isolation of patients with confirmed RIF-resistance, and referral to a designated drug-resistant TB care centre.

Data collection

A list of patients who were $>14$ years old, and had RIF-resistant MTB confirmed by an Xpert MTB/RIF assay, was compiled and their records were obtained from the CHBAH's records department. Data from the NHLS database and the TB care centre at CHBAH were obtained to support information obtained from the patients' hospital records. For patients with confirmed RIF-resistance, a name search was performed on the NHLS database to obtain all confirmatory sputum samples that were submitted. The data collection sheet used for the study recorded patient demographics, HIV status, CD4 count, antiretroviral therapy (ART), previous TB exposure, previous TB treatment completion, current anti-TB drug treatment, the time taken to obtain the MTB/RIF assay result, the submission and results of additional TB susceptibility tests, isolation of the patient, appropriate referral and hospital outcome. The data were then collated and entered on an Excel worksheet.

Permission to conduct this study was granted by the Human Research Ethics Committee at the University of the Witwatersrand (ref. no. M131033) and the Medical Advisory Committee at CHBAH. The National Manager of Academic Affairs and Research at the NHLS granted permission to access their result database.

\section{Results}

Prevalence of RIF-resistant TB

Of the 729 positive Xpert MTB/RIF assays submitted, 77 were positive for RIF resistance. The prevalence of RIF-resistant PTB among patients who presented to CHBAH between March 2011 and February 2013, was $10.6 \%$. Seven patients' files could not be traced and therefore they were not included in further analysis. Of the 70 patients, $22(31.4 \%)$ had RMR-TB, 19 (27.1\%) had MDR-TB, and 29 (41.4\%) patients had no other confirmatory test submitted and therefore the pattern of drug resistance could not be established.

Demographic characteristics, HIV co-infection and TB history Thirty-four patients (49\%) were female. The mean (SD) age was 36 (10.2) years. Among 66 patients with known HIV-serostatus, 58 (83\%) patients were HIV-positive and 8 (11\%) patients were HIVnegative. Among the 51 (88\%) patients who had their CD4 cell counts recorded, the mean $\mathrm{CD} 4$ cell count was 91 cells $/ \mu \mathrm{L}$. The median $\mathrm{CD} 4$ cell count was 43 cells $/ \mu \mathrm{L}$ (interquartile range (IQR) 15 - 160). Thirtytwo (55\%) HIV-positive patients were on ART prior to admission. Forty-one (59\%) patients had a history of previous TB, among whom $11(26.8 \%)$ patients had more than one episode of TB. Four (9.8\%) patients admitted to not completing their prescribed treatment regimen previously. Twenty-six (37\%) patients were being treated for susceptible PTB at the time of hospitalisation (Table 1).

\section{Management}

Of the 70 patients with RIF-resistant TB, 12 (17\%) patients had been discharged and 5 (7\%) patients had died. Therefore 53 (75.7\%) patients were still in hospital when their Xpert MTB/RIF result was obtained. Table 2 displays the results of the management outcomes for the total cohort $(N=70)$ and the subgroup $(N=53)$ of patients who received their results in hospital.

\section{Additional susceptibility testing}

It took on average 3.36 days (range: 1 - 10 days) from the date of admission to obtain the Xpert MTB/RIF result (Table 2). Overall, $42(60 \%)$ patients had the LPA test submitted, to differentiate 


\begin{tabular}{|c|c|}
\hline & $n(\%) *$ \\
\hline \multicolumn{2}{|l|}{ Prevalence } \\
\hline RMR & $22(3.02)$ \\
\hline MDR-TB & $19(2.60)$ \\
\hline Unclassified & $29(3.97)$ \\
\hline \multicolumn{2}{|l|}{ Baseline characteristics } \\
\hline Female & $34(49)$ \\
\hline Age (years), mean (SD) & $36(10.2)$ \\
\hline HIV-positive & $58(83)$ \\
\hline CD4 cell count (cells/ $\mu \mathrm{L})$, median (IQR) & $43(15-160)$ \\
\hline CD 4 cell count $<100$ cells $/ \mu \mathrm{L}$ & $37(73)$ \\
\hline ART & $32(55)$ \\
\hline Previous history of TB infection & $41(59)$ \\
\hline Discharged before results available & $12(17)$ \\
\hline $\begin{array}{l}\text { Time to obtain Xpert MTB/RIF assay } \\
\text { result (days), mean (SD) }\end{array}$ & $3.36(2.28)$ \\
\hline $\begin{array}{l}\text { Duration of hospitalisation (days), } \\
\text { median (IQR) }\end{array}$ & $11(6-18)$ \\
\hline Inpatient mortality & $13(19)$ \\
\hline \multicolumn{2}{|c|}{$\begin{array}{l}\mathrm{TB}=\text { tuberculosis; } \mathrm{RMR}=\text { rifampicin mono-resistance; } \mathrm{MDR}-\mathrm{TB}=\text { multi-drug resistant } \\
\text { tuberculosis; } \mathrm{SD}=\text { standard deviation; } \mathrm{ART}=\text { antiretroviral therapy; } \mathrm{IQR}=\text { interquartile range } \\
\mathrm{MTB} / \mathrm{RIF}=\text { rifampicin-resistant } \text { Mycobacterium tuberculosis. }\end{array}$} \\
\hline
\end{tabular}

Table 2. Management outcomes for study cohort $(N=70)$ and remaining inpatients $(N=53)^{\star}$

\begin{tabular}{lll}
\hline & \multicolumn{2}{c}{$\boldsymbol{n ( \% )}$} \\
\cline { 2 - 3 } Outcome & $\begin{array}{l}\text { Study cohort } \\
(\mathbf{N = 7 0 )}\end{array}$ & $\begin{array}{l}\text { Inpatients } \\
(\mathbf{N = 5 3 )}\end{array}$ \\
\hline LPA & $42(60)$ & $38(72)$ \\
DST & $18(26)$ & $16(30)$ \\
TB culture & $42(60)$ & $36(68)$ \\
TB microscopy & $53(76)$ & $46(87)$ \\
No additional testing & $20(29)$ & $11(21)$ \\
Adequate drug treatment & $23(33)$ & $23(43)$ \\
Patients isolated & $35(50)$ & $35(66)$ \\
Patients appropriately referred & $34(49)$ & $34(64)$ \\
LPA = line probe assay; DST = drug susceptibility testing; TB = tuberculosis. \\
*Management outcomes of 70 patients with rifampicin-resistant TB and the 53 patients still \\
hospitalised on receipt of results.
\end{tabular}

between MDR-TB and RMR-TB. Of the sputum samples submitted, $53(76 \%)$ and $42(60 \%)$ patients had submitted sputum specimens for TB microscopy and TB culture, respectively. Eighteen (26\%) patients had submitted sputum samples for DST. Of the 53 patients who received results in hospital, 38 (72\%) patients had the LPA test, $46(87 \%)$ and $36(68 \%)$ patients had a TB microscopy and TB culture sent, respectively. DSTs were requested for $16(30 \%)$ patients. Twenty (29\%) patients of the overall cohort had no further sputum tests requested.

\section{Drug therapy}

Of the anti-TB drug therapy that was provided to patients, 23 were initiated on appropriate treatment for MDR-TB, representing $33 \%$ of the total cohort and $43 \%$ of the 53 remaining inpatients (Table 2). Thirty-nine patients were kept on treatment for drug-susceptible TB.

\section{Isolation, referral and outcome}

It took an average of 4 days to isolate patients. The median duration of hospitalisation was 11 days (IQR 6 - 18). Half of the patients in the study cohort and $66 \%$ of the inpatients were isolated, respectively. Appropriate referral was carried out for 34 patients, constituting $49 \%$ of the cohort and $64 \%$ of the 53 remaining patients.

Of the other 36 patients, 10 (14\%) patients were not appropriately referred, 12 (17\%) patients were discharged before their result became available, 1 (1.4\%) absconded, and 13 (19\%) patients died in hospital.

\section{Discussion}

Study of the initial inpatient management of patients diagnosed with RIF-resistant PTB is important for two main reasons. Firstly, for the patient's benefit, it measures the time to diagnosis after hospitalisation, the submission of further sputum tests to define the extent of resistance to anti-TB drugs, early initiation of guideline-recommended therapy, and referral for supervision and management. Secondly, for the purpose of infection control to protect other patients and healthcare workers, it investigates patient isolation and initiation of effective treatment. Knowing the prevalence of RIF-resistant TB can strengthen the case for effective infection control in a given population.

The prevalence of RIF-resistance during the 2 -year period was $10.6 \%$. This was more than double the rate of $4.6 \%$ found in a national survey conducted in SA during the period $2012-2014 .{ }^{[32]}$ The prevalence of RMR (3.02\%) exceeded that of MDR-TB (2.60\%), but the unclassified group (3.97\%) was the largest due to a lack of submission of confirmatory tests. Since CHBAH is a referral centre for patients with complicated and non-resolving disease processes, it could be expected that the prevalence rates would be higher than the national average. The prevalence rate of RMR has doubled in new TB cases nationally, and in the Western Cape Province, since 2002. ${ }^{[32,33]}$ Risk factors for the increase in prevalence were advanced HIV infection and previous TB treatment. ${ }^{[33]}$

The recommended management strategy of confirmed RIF-resistant TB includes submission of confirmatory tests, initiation of MDRTB therapy, isolation, and referral to an appropriate health facility to continue management. ${ }^{[27]}$ The confirmatory tests include sputum samples sent for TB microscopy and culture, LPA, and DST. Overall, our study revealed that $76 \%, 60 \%, 60 \%$ and $26 \%$ of TB microscopy, TB culture, LPA and DST samples were submitted, respectively. A likely reason for the disjuncture between rates of submission for culture and DST is that the laboratory form requires the clinician to request DST separately from culture submissions, whereas many clinicians assume that susceptibility tests would be performed automatically. Two factors that may have contributed negatively to the overall results were patients who were discharged (17\%) or had died (7\%) before their result had become available. After these two 
factors had been taken into consideration, $72 \%$ had the LPA test, $68 \%$ had the TB culture submitted, and $30 \%$ had the DST requested, which still demonstrates a substantial deficiency in the submission of confirmatory tests despite having a confirmed result on MTB/RIF assay. In Zimbabwe, the submission rate of sputum samples for culture and DST at two healthcare centres was 70\%, of which only half reached the laboratory and were processed. ${ }^{[34]}$ Possible explanations for our results are that patients may have been transferred to the referral centre before a confirmatory specimen was submitted, the patient may no longer have been expectorating sputum, or the attending doctor may not have known the recommended protocol. Premature discharging of patients is a direct result of limited hospital beds and a high patient demand at our centre. ${ }^{[2]}$ Patient tracing and continued surveillance would ensure that these patients receive the diagnosis and appropriate medical treatment, despite being discharged early. Educating healthcare workers and encouraging sputum sample collection immediately after obtaining the result, can improve the rate of confirmatory tests submission and thus ensure accurate diagnosis and treatment. A better solution would be to send paired samples, whereby the laboratory would retain the second sputum sample for the supplementary and confirmatory tests, which would be triggered once the MTB/RIF assay detects RIF resistance.

A fundamental part of managing drug-resistant TB is the correct combination of therapeutic drugs. At the time of the study being conducted, the five essential drugs used during the intensive phase to treat MDR-TB were kanamycin or amikacin, moxifloxacin, ethionamide, terizidone, or cycloserine and pyrazinamide. ${ }^{[27]}$ In our high-prevalence setting, the presence of RIF resistance may be used as a surrogate for MDR-TB; however due to the recent studies displaying an increase in RMR prevalence, ${ }^{[32-33]}$ this may no longer be true. Nonetheless, each of the 70 patients from our study should have been initiated on MDR-TB treatment. The study revealed that $<50 \%$ of the patients received appropriate drug treatment in hospital. Of the patients who were inadequately treated, $89 \%$ were kept on treatment for drug-susceptible TB. The regimen may not have been changed due to the patient being prematurely discharged (17\%) or dying (7\%) before their result became available, or possibly due to the patient being rapidly transferred to a dedicated centre for drug-resistant TB management. Inadequate knowledge of the appropriate drug regimen by the treating doctors may have accounted for the reluctance to amend treatment. It is imperative that all healthcare providers that are faced with the challenge of TB and drug-resistance on a day-to-day basis become familiar with the appropriate drug-resistant treatment regimen.

It took an average of 3.36 days from the day of admission to confirm the presence of RIF-resistant PTB. The Xpert MTB/RIF assay was introduced for use as a point-of-care, rapid diagnostic assay in poor rural areas. Its use in the tertiary level hospital revealed a longer duration before obtaining the result, which was contrary to the intended rapid use of the assay. A delay in obtaining the result leads to a delay in appropriate treatment, and exposure of the hospital population to drug-resistant organisms. Possible explanations include a delay in obtaining a sputum sample from the patient, delays in transport, registration, or processing of the specimen by the laboratory, and sputum samples that may be lost in transit. ${ }^{[34]}$ A contemporary investigation of the time to obtaining a result would be beneficial. After patients who were discharged or had died before their result became available were taken into account, $66 \%$ of the remaining 53 patients were isolated with a mean time of 4 days after being admitted. The time taken to isolation corresponds to the duration of time taken to obtain the MTB/RIF assay result. Appropriately isolating patients with drug-resistant TB, while awaiting referral, will decrease the risk of further transmission in the hospital setting. Many hospitals have a limited number of isolation facilities, which are typically reserved for patients with a high clinical suspicion for, or confirmed, drugresistant TB.

Appropriate referral forms an essential part of the management of drug-resistant $\mathrm{TB}$. The referral centre will: ensure that patients have the correct confirmatory tests submitted and will review the results; prescribe correct drug therapy and will have adequate stock of drugs; screen for HIV and initiate and manage ART; monitor progress with resubmission of sputum specimens for testing to assess treatment success; manage adverse drug reactions; and have access to allied healthcare providers to discuss dietary advice, to manage psychological issues, and to perform hearing tests for those on aminoglycosides. Of the 53 patients who received their results in hospital, $64 \%$ were appropriately referred, while $14 \%$ were not referred, with the remainder dying in hospital, and a single case absconded.

The mortality rate was $19 \%$, which was comparable with the global estimate of $16 \%$ among patients with MDR-TB ${ }^{[4]}$ and a local study ${ }^{[29]}$ that demonstrated an inpatient mortality rate of $19 \%$ among patients diagnosed with PTB. A delay in obtaining the diagnosis, inappropriate drug therapy, and advanced HIV disease may have contributed to the overall mortality.

The prevalence of HIV and any RIF-resistant TB co-infection in this cohort was $83 \%$, which is in keeping with global comparisons of $70-80 \%,,^{[2,14]}$ and corresponds with the high levels found in subSaharan Africa. Ghandhi et al.$^{[18]}$ demonstrated a rate of $92 \%$ with MDR-TB and HIV co-infection. Most of the patients had advanced immunosuppression.

\section{Study limitations}

Owing to the lack of submission of the follow-up LPA and DST tests, a considerable proportion (40\%) of the study population could not be assigned a specific resistance pattern. Although the NHLS system was searched by name for evidence of follow-up testing, the retrospective nature of the study did not allow us to trace patients who had not submitted tests, to ascertain details of further testing and appropriate care. Although the study design allowed for the description of prevalence and initial management at the beginning of the treatment programme, it did not incorporate patients' adherence to treatment, adverse reactions, and long-term outcomes. The study only reviewed a single healthcare facility and therefore the results only pertain to a limited geographical area, which may not be fully representative of other regions in SA. The adequacy of records and untraceable records was another limitation to the study. The quality of information recorded within the inpatient hospital files was sufficient in most instances.

\section{Conclusion}

In conclusion, our results revealed that the prevalence of RIFresistant PTB in our setting was $>10 \%$. The study also showed that the overall management (including confirmatory tests, appropriate drug therapy, isolation, and referral) of patients with RIF-resistant 
PTB was substandard and there are multiple areas that need to be improved upon. Submission of paired sputum samples and storage by the laboratory may improve the diagnostic yield of drug-resistant TB. Educating healthcare professionals regarding initial management and therapeutic regimens is of paramount importance to help control the scourge of this treatable disease.

Acknowledgements. Mr Tinyiko Ngobeni, health data analyst at the Corporate Data Warehouse for the National Health Laboratory Service, who compiled the list of Xpert MTB/RIF assays performed during the study period. Chris Hani Baragwanath Academic Hospital records office, for tracing of patients' files. Honest Muchabaiwa, for his assistance regarding the overall data interpretation.

Author contributions. Design, analysis and interpretation - RMN, ASK, FS. Drafting and revision - RMN, ASK, FS. Approval of version to be published - RMN, ASK, FS.

Funding. None.

Conflicts of interest. None

1. World Health Organization. Global Tuberculosis Report. Geneva: WHO, 2015. http:// apps.who.int/iris/bitstream/10665/191102/1/9789241565059_eng.pdf (accessed 27 July 2016).

2. World Health Organization. Global tuberculosis report. Geneva: WHO, 2014. http:// apps.who.int/iris/bitstream/10665/137094/1/9789241564809_eng.pdf (accessed on 24 March 2015).

3. Kalokhe AS, Shafiq M, Lee JC, et al. Multidrug-resistant tuberculosis drug susceptibility and molecular diagnostic testing. Am J Med Sci 2013;345(2):143-148. http://doi.org/10.1097/MAJ.0b013e31825d32c6

4. World Health Organization. Drug resistant TB surveillance \& response supplement, Global Tuberculosis Report. Geneva: WHO, 2014. www.who.int/tb/publications/ global_report/gtbr14_supplement_web_v3.pdf (accessed on 24 March 2015).

5. Caminero JA. Multidrug-resistant tuberculosis: Epidemiology, risk factors and case finding. Int J Tuberc Lung Dis 2010;14(4):382-390.

6. Ormerod LP. Multidrug-resistant tuberculosis (MDR-TB): Epidemiology, prevention and treatment. Br Med Bull 2005;73-74(1):17-24. http://doi.org/10.1093/bmb/ldh047

7. Shamaei M, Marjani M, Chitsaz E, et al. First-line anti-tuberculosis drug resistance patterns and trends at the national TB referral center in Iran - eight years of surveillance. Int J Infect Dis 2009;13(5):e236-e240. http://doi.org/10.1016/j. ijid.2008.11.027

8. Sandman L, Schluger NW, Davidow AL, Bonk S. Risk factors for rifampinmonoresistant tuberculosis: A case-control study. Am J Respir Crit Care Med 1999;159(2):468-472. http://doi.org/10.1164/ajrccm.159.2.9805097

9. Nakiyingi L, Nankabirwa, H, Lamorde M. Tuberculosis diagnosis in resource-limited settings: Clinical use of GeneXpert in the diagnosis of smear-negative PTB: A case report. Afr Health Sci 2013;13(2):522-524. http://doi.org/10.4314/ahs.v13i2.46

10. Meyssonnier V, Bui TV, Veziris N, Jarlier V, Robert J. Rifampicin mono-resistant tuberculosis in France: A 2005-2010 retrospective cohort analysis. BMC Infect Dis 2014;14:18. https://doi.org/10.1186/1471-2334-14-18

11. Keugoung B, Fouelifack FY, Fotsing R, Macq J, Meli J, Criel B. A systematic review of missed opportunities for improving tuberculosis and HIV/AIDS control in Subsaharan Africa: What is still missed by health experts? Pan Afr Med J 2014;18:320. http://doi.org/10.11604/pamj.2014.18.320.4066

12. World Health Organization. Global update on the health sector response to HIV. Geneva: WHO, 2014. http://apps.who.int/iris/bitstream/10665/128494/1/9789241507585_eng. pdf?ua $=1$ (accessed on 19 May 2015)

13. Dean AS, Zignol M, Falzon D, Getahun H, Floyd K. HIV and multidrug-resistant tuberculosis: Overlapping epidemics. Eur Respir J 2014;44(1):251-254. http://doi. org/10.1183/09031936.00205413

14. Wells CD. Global impact of multidrug-resistant pulmonary tuberculosis among HIV-infected and other immunocompromised hosts: Epidemiology, diagnosis, and strategies for management. Curr Infect Dis Rep 2010;12(3): 192-197. http://doi. org/10.1007/s11908-010-0104-5
15. Farley JE, Ram M, Pan W, et al. Outcomes of multi-drug resistant tuberculosis (MDRTB) among a cohort of South African patients with high HIV prevalence. PLoS ONE 2011;6(7):e20436. http://doi.org/10.1371/ journal.pone.0020436

16. Chung-Delgado K, Guillen-Bravo S, Revilla-Montag A, Bernabe-Ortiz A. Mortality among MDR-TB cases: Comparison with drug-susceptible tuberculosis and associated factors. PLoS ONE 2015;10(3):e0119332. http://doi.org/10.1371/journal. pone. 0119332

17. Van den Hof S, Tursynbayeva A, Abildaev T, Adenov M, Pak S, Ismailov S. HIV and multidrug-resistant tuberculosis: Overlapping risk factors. Eur Respir J 2015;45(2):567-569. http://doi.org/10.1183/09031936.00131014

18. Gandhi NR, Andrews JR, Brust JC, et al. Risk factors for mortality among MDRand XDR-TB patients in a high HIV prevalence setting. Int J Tuberc Lung Dis 2012;16(1):90-97. http://doi.org/10.5588/ijtld.11.0153.

19. Traore H, Fissette K, Bastian I, Devleeschouwer M, Portaels F. Detection of rifampicin resistance in Mycobacterium tuberculosis isolates from diverse countries by a commercial line probe assay as an initial indicator of multidrug resistance. Int $\mathrm{J}$ Tuberc Lung Dis 2000;4(5):481-484.

20. Sougakoff W, Rodrigue M, Truffot-Pernot C, et al. Use of a high-density DNA probe array for detecting mutations involved in rifampicin resistance in Mycobacterium tuberculosis. Clin Microbiol Infect 2004;10(4):289-294. https://doi.org/10.1111/ j.1198-743X.2004.889.x

21. Kurbatova EV, Cavanaugh JS, Shah NS, et al. Rifampicin-resistant Mycobacterium tuberculosis: Susceptibility to isoniazid and other anti-tuberculosis drugs. Int J Tuberc Lung Dis 2012;16(3):355-357. http://doi.org/10.5588/ijtld.11.0542

22. World Health Organization. Global Tuberculosis Report. Geneva: WHO, 2012. http:// apps.who.int/iris/bitstream/10665/75938/1/9789241564502_eng.pdf (accessed 03 March 2013).

23. Theron G, Peter J, van Zyl-Smit R, et al. Evaluation of the Xpert MTB/RIF assay for the diagnosis of pulmonary tuberculosis in a high HIV prevalence setting. Am J Respir Crit Care Med 2011;184(1):132-140. http://doi.org/10.1164/rccm.201101-0056OC

24. Steingart KR, Sohn H, Schiller I, Kloda LA, Boehme CC, Pai M, Dendukuri N. Xpert MTB/RIF assay for pulmonary tuberculosis and rifampicin resistance in adults. Cochrane Database Syst Rev 2014;1(1):CD009593. http://doi.org/10.1002/14651858. CD009593.pub3

25. Baker BJ, Holtom PD. Additional benefits of GeneXpert MTB/RIF assay for the evaluation of pulmonary tuberculosis among inpatients. Clin Infect Dis 2015;60(8):1287-1288. http://doi.org/10.1093/cid/civ006

26. Weyer K, Mirzayev F, Migliori G, et al. Rapid molecular TB diagnosis: Evidence, policy-making and global implementation of Xpert(R)MTB/RIF. Eur Respir J 2013;42(1):252-271. http://doi.org/10.1183/09031936.00157212

27. Department of Health, Republic of South Africa. Management of drug-resistant tuberculoisis policy guidelines. Pretoria: Department of Health, 2011.

28. Van Altena R, de Vries G, Haar CH, et al. Highly successful treatment outcome of multidrug-resistant tuberculosis in the Netherlands, 2000-2009. Int J Tuberc Lung Dis 2015;19(4):406-412. http://doi.org/10.5588/ijtld.14.0838

29. Edginton ME, Wong ML, Phofa R, Mahlaba D, Hodkinson HJ. Tuberculosis at Chris Hani Baragwanath Hospital: Numbers of patients diagnosed and outcomes of referrals to district clinics. Int J Tuberc Lung Dis 2005;9(4):398-402.

30. Ebonwu JI, Tint KS, Ihekweazu C. Low treatment initiation rates among multidrug resistant tuberculosis patients in Gauteng, South Africa, 2011. Int J Tuberc Lung Dis 2013;17(8):1043-1048. http://doi.org/10.5588/ijtld.13.0071

31. Nkosi D, Janssen S, Padanilam X, Louw R, Menezes CN, Grobusch MP. Factors influencing specialist care referral of multidrug- and extensively drug-resistant tuberculosis patients in Gauteng/South Africa: A descriptive questionnaire-based study. BMC Health Serv Res 2013;13:268. http://doi.org/10.1186/1472-6963-13-268

32. National Institute for Communicable Diseases. South African Tuberculosis Drug Resistance Survey 2012-2014, Sandringham, South Africa, http://www.nicd.ac.za (accessed 21 November 2016).

33. Mukinda FK, Theron D, van der Spuy GD, et al. Rise in rifampicin-monoresistant tuberculosis in Western Cape, South Africa. Int J Tuberc Lung Dis 2012;16(2):196202. http://doi.org/10.5588/ijtld.11.0116

34. Charambira K, Ade S, Harries AD, et al. Diagnosis and treatment of TB patients with rifampicin resistance detected using Xpert ${ }^{\circ}$ MTB/RIF in Zimbabwe. Public Health Action 2016;6(2):122-128. http://doi.org/10.5588/pha.16.0005 